\title{
Experimental measurement of picometer scale spontaneous vibrations in a precision deployable boom under thermal loading ${ }^{1}$
}

\author{
Mark J. Silvera,*, Lee D. Peterson ${ }^{\mathrm{b}}$ and Lisa M.R. Hardaway ${ }^{\mathrm{c}}$ \\ a Structures and Transportation Group, Foster-Miller Inc., Waltham, MA, USA \\ ${ }^{\mathrm{b}}$ University of Colorado, Center for Aerospace Structures, Department of Aerospace Engineering Sciences, \\ Boulder, CO, USA \\ ${ }^{\mathrm{c}}$ Ball Aerospace, Boulder, CO, USA
}

Received 19 July 2004

Revised 7 June 2006

\begin{abstract}
This paper reports observations and analysis of picometer scale spontaneous vibrations in a precision deployable boom under thermal loading. The structural test article is a deployable boom previously flown in space. It exhibited spontaneous vibrations during the temperature rise following a night to day transition on orbit. In an attempt to reproduce the spontaneous vibrations on the ground, the test article was thermally loaded within a mechanically stabilized test environment. Spontaneous vibrations were induced in these ground experiments. The vibrations were at a scale of motion for which current theories would not expect such a release. The amplitudes of these vibrations were on the order of a few dozen picometers, and the frequency was near $1500 \mathrm{~Hz}$. Evidence of wave dispersion was detected in the vibrations.
\end{abstract}

\section{Introduction}

Structures are designed to hold a specific shape under specified load conditions. For space-based telescopes, the structural shape stability under orbital loads must be maintained to within a small fraction of a wavelength of light. Requirements for the Next Generation Space Telescope (NGST) would have required dynamic stability of the mirror petals to within a few nanometers [3]. Interferometric telescopes, such as the Space Interferometry Mission (SIM), require this level of stability or better, but over a bandwidth of perhaps $1000 \mathrm{~Hz}$ [5]. These requirements are necessary if the structural imperfection is to be kept compatible with the active optical elements of the telescope.

Little is available in the literature about mechanics and stability at the nanometer scale of motion. Micrometer level instabilities in materials and structures have been recognized for over 100 years [2], yet data at the nanometer level have been a relatively recent development. High bandwidth instruments now exist that can measure nanometer level displacements and micro gravity level accelerations. The use of these instruments has resulted in a small but growing number of experiments on the behavior of structures at these scales [7-10].

The present study provides additional evidence in this regard. The objective is to measure and characterize the stability of a deployed boom under thermal loading. It is well known at both the macroscopic and microscopic scales of motion that thermo elastic loading of a spacecraft structure can induce spontaneous vibrations. In the first

\footnotetext{
${ }^{1}$ Based on paper number AIAA-2001-1306, which appeared in the proceedings of the 42nd Structures, Structural Dynamics and Materials Conference, Seattle, Washington, April, 2001.

*Corresponding author. Tel.: +1 781684 4031; Fax: +1 781684 4410; E-mail: msilver@foster-miller.com.
} 
set of Hubble Space Telescope (HST) solar arrays, for example, thermally-induced bending caused a well-known pointing jitter [16]. Analysis of the jitter problem showed that thermally induced bending in the Bi-STEM structural supports for the solar arrays resulted from temperature changes when HST passed from shadow to sunlight and vice versa. There were two distinct types of thermally induced vibrations seen in the Hubble solar arrays, the disturbances occurring during the orbital days and eclipse/sunlight transitions and the disturbances occurring during the sunlight/eclipse transitions. The former happened when the thermally induced bending loads exceeded the Coulombic friction threshold in the Bi-STEM, causing a sudden loss of stiffness and a resulting release of strain energy [6]. Such phenomena are well recognized among the spacecraft structural dynamics community.

Perhaps what may be little appreciated is that the trigger of such frictional instabilities is associated theoretically with pre-slip deformations on the order of microns, and with critical velocities on the order of microns per second or more [1]. According to accepted dynamic friction theory, unless the load and load rate causes the frictional interface to approach both these critical thresholds there should not be an induced vibration. In such a case, a jointed structure should retain its stability undergoing at most progressive microslip shearing in its friction interfaces [11]. Said another way, if gross slip is not induced in the friction interfaces in such a way to create an unstable dynamic stiffness, there should be no sudden release of strain energy. One could simply design structures to avoid this limit. However, this expectation is based on models derived entirely from empirical evidence gathered at the macroscopic and microscopic deformation scale. Space telescopes need to be stable at the nanometer scale (perhaps below). With no data at this scale of motion, exploratory experiments are needed to test the limits of accepted theory.

To this end, the NASA Jet Propulsion Laboratory (JPL) performed flight tests on a deployed boom in the Interferometry Program Experiment 2 (IPEX-2). One objective of IPEX-2 was to detect and characterize any spontaneous vibrations that might be induced by thermal loads in a space environment. The results of the flight test were presented in Reference [10].

Perhaps the most puzzling data was collected immediately following a particular night to day transition. As the truss was heated, the temperature rose approximately 30 degrees $C$. Many spontaneous vibrations were recorded at this time. Following the flight, exploratory testing at JPL indicated that these low amplitude thermal snaps might have been observed on the ground during one of the post-flight experiments [12]. A subsequent thermo elastic analysis at JPL indicated that the loads generated during flight should have remained below the Coulombic level, although there was some ambiguity as to whether the loads in the mounting hardware exceeded the stick-slip threshold [14].

At about the same time, Reference [9] provided data on spontaneous vibrations in a different, sub-scale model truss in $1 \mathrm{~g}$ testing. Heating the truss slowly while suspended in both convective and radiative heat chambers apparently induced these vibrations. In the convective heating chamber, accelerations were observed with amplitudes between 0.1 and $1 \mathrm{~g}$ and with frequencies between 1 and $18 \mathrm{kHz}$. Radiative heating induced spontaneous vibrations between 1 and 5 gees with frequencies between 9 and $18 \mathrm{kHz}$. These vibrations seemed to be limited in extent to local regions of the structure. Although there was no analysis of the induced loads produced in these experiments, the amplitudes of motion were estimated to be at the nanometer scale, and may therefore be evidence of thermally induced vibrations in a microslip load regime; however, this remained an open question.

Experiments reported in $[7,8]$ tested the nanometer scale stability of a single bay of the IPEX-2 boom. Those experiments reported spontaneous vibrations induced by mechanical cycling of the bay in shear. The vibrations were correlated with the magnitude of entrapped strain energy stored in the structure by friction during the cycling. A design methodology for these mechanically induced spontaneous vibrations was also reported in [15].

The experiments reported in this paper were motivated by the question as to whether a thermal load could also produce small scale spontaneous vibrations, as the mechanical experiments were able to do. These experiments were able to successfully induce vibrations by heating the region of one bay of the truss near the root. Several picometer scale spontaneous vibrations were measured. Moreover, the vibrations show evidence of wave transmission and dispersion down the boom away from the area of the applied thermal load.

This paper is organized as follows: The first section presents the experimental apparatus and procedure including the configuration, test measurements and data acquisition. The second section summarizes the results of the tests including time and frequency domain characterization. The third section is a discussion of possible exogenous vibration sources, and reasons why they can be eliminated as sources of the spontaneous vibrations seen in these tests. 


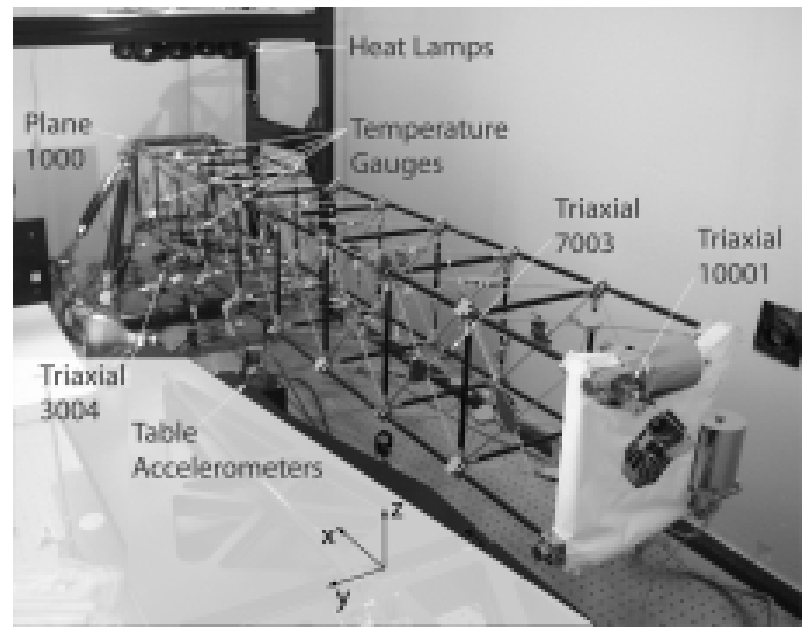

Fig. 1. IPEX-2 Test Configuration.

\section{Experimental apparatus and procedure}

\subsection{Configuration}

The IPEX boom was mounted on an isolated optics table in the Thermally and Acoustically Stabilized Chamber (TASC). This chamber passively isolates the experiments inside from ground vibration, acoustics, and thermal variations. Typical table vibrations of 5 micro-gravities RMS are achieved over a $1000 \mathrm{~Hz}$ bandwidth [7].

The test configuration is shown in Fig. 1. The Boom was mounted inside the TASC on the optics table using the kinematic mounts used in the flight experiment. These mounts can be seen in the figure at the far end of the boom. This end of the boom was designated Plane 1000. Five 200-watt heat lamps were suspended above the boom directly above the first bay, between Plane 1000 and 2000. Prior analysis indicated that this region of the structure was most likely suspected in the generation of the spontaneous vibrations on orbit [14]. The heat lamps were at a distance of 11.5 inches from the boom to the tips of the bulbs. They were arranged in a 10-inch by 12-inch rectangular layout with the fifth bulb at the middle of the rectangle. The lamps were held in a structure attached to the chamber floor, not connected to the optics bench.

\subsection{Test measurements}

Table 1 provides a detailed list of the test measurements used in this experiment. The temperature and vibration of the boom and the test environment were measured before, during and after thermal loading. The temperature was measured by four temperature gauges placed in the center of the battens at plane 2000. Triaxial accelerometers were placed at three other batten planes. The first of these was placed at batten plane 3000, which was one bay away from the thermally loaded bay. Other triaxial accelerometers were placed at plane 7000, near the middle of the boom, and plane 10000, the tip of the boom.

The primary environmental sensors were the seismic accelerometers that monitored the vibration of the optics bench. These sensors were mounted on the optics bench in a 6 DOF arrangement just below the mid-span of the boom. This is shown in Fig. 1.

Table 1 also shows the bandwidth and broadband noise of the sensors. The micro-g resolution and kilohertz bandwidth provided sufficient sensitivity to detect vibrations in the nanometer scale, and perhaps below at high frequency. 
Table 1

Test Measurements

\begin{tabular}{lllllc}
\hline Name & Type & Location & Direction & Bandwidth & Noise $(\mu \mathrm{g})$ \\
\hline Table Vibration & Accelerometer & 101 & $+\mathrm{X}$ & $500 \mathrm{~Hz}$ & 1 \\
Table Vibration & Accelerometer & 101 & $+\mathrm{Y}$ & $500 \mathrm{~Hz}$ & 1 \\
Table Vibration & Accelerometer & 101 & $+\mathrm{Z}$ & $500 \mathrm{~Hz}$ & 1 \\
Table Vibration & Accelerometer & 101 & $+\mathrm{X}$ & $2.5 \mathrm{kHz}$ & 5 \\
Table Vibration & Accelerometer & 101 & $+\mathrm{Y}$ & $2.5 \mathrm{kHz}$ & 5 \\
Table Vibration & Accelerometer & 101 & $+\mathrm{Z}$ & $2.5 \mathrm{kHz}$ & 5 \\
IPEX Boom Vibration & Accelerometer & 10001 & $+\mathrm{X}$ & $5 \mathrm{kHz}$ & 60 \\
IPEX Boom Vibration & Accelerometer & 10001 & $+\mathrm{Y}$ & $5 \mathrm{kHz}$ & 60 \\
IPEX Boom Vibration & Accelerometer & 10001 & $+\mathrm{Z}$ & $5 \mathrm{kHz}$ & 60 \\
IPEX Boom Vibration & Accelerometer & 7003 & $+\mathrm{X}$ & $5 \mathrm{kHz}$ & 60 \\
IPEX Boom Vibration & Accelerometer & 7003 & $+\mathrm{Y}$ & $5 \mathrm{kHz}$ & 60 \\
IPEX Boom Vibration & Accelerometer & 7003 & $+\mathrm{Z}$ & $5 \mathrm{kHz}$ & 60 \\
IPEX Boom Vibration & Accelerometer & 3004 & $+\mathrm{X}$ & $5 \mathrm{kHz}$ & 60 \\
IPEX Boom Vibration & Accelerometer & 3004 & $+\mathrm{Y}$ & $5 \mathrm{kHz}$ & 60 \\
IPEX Boom Vibration & Accelerometer & 3004 & $+\mathrm{Z}$ & $5 \mathrm{kHz}$ & 60 \\
IPEX Boom Temp & IC Temperature & 2034 & Scalar & & \\
IPEX Boom Temp & IC Temperature & 2012 & Scalar & & \\
IPEX Boom Temp & IC Temperature & 2023 & Scalar & & \\
IPEX Boom Temp & IC Temperature & 2041 & Scalar & \\
H & Humidity Sensor & 102 & Scalar & & \\
T & Temperature & 102 & Scalar & & \\
P & Barometer & 102 & Scalar & & \\
\hline
\end{tabular}

\subsection{Data acquisition}

Data was acquired using a high-speed transient digitizer and recorded to disk for subsequent analysis. The sample rate of the digitizer was kept at $5000 \mathrm{~Hz}$ for each test. All sensors were filtered with an analog anti-aliasing filter set so that responses above the Nyquist frequency were less than the bit resolution of the digitizer.

Data was collected before, during, and after thermal load application. Each data window, nearly 5 Mbytes of data, was approximately 13 seconds long. Data was recorded to disk for approximately another 20 seconds, then data acquisition was immediately continued. This meant that the data duty cycle of the data acquisition was approximately $40 \%$.

\subsection{Test procedure}

Because of the very low levels of motion expected during the testing, all experiments were conducted during the nighttime hours when building and traffic noise did not interrupt the experiments. Background vibration during this period typically falls to less than 10 microgravities RMS, over the entire bandwidth of the sensors. This is due to both the use of a damped, isolated optical bench and an enclosed acoustic shield, which also provided a thermal insulator. Thermal stability of better than 10 milli-degrees Kelvin for several hours was also typically observed, but only after allowing the test environment to passively stabilize for at least 12 hours (preferably 24 hours) after closure.

Each test began after long-term thermal stability had been achieved. Then, the heat lamps were turned on for a period of 5 minutes. Once lamp power was turned off, the test article was allowed to passively cool before the next thermal load was applied. In a particular night, three thermal loads could be applied with approximately 1 hour of thermal re-stabilization between them.

\subsection{Data analysis protocols}

The systematic detection of a spontaneous vibration from data such as this remains a subject for research. The challenge is to identify a short-lived transient from a high bandwidth data set. Pixelation in a time domain plot of this kind of data makes direct visual detection unreliable. For this reason, this study used the methods described in [7]. 
Table 2

Detected Spontaneous Vibrations

\begin{tabular}{|c|c|c|c|c|c|c|c|}
\hline Event & $\mathrm{t}(\mathrm{sec})$ & $\Delta \mathrm{T}\left({ }^{\circ} \mathrm{C}\right)$ & Position & Direction & Acceleration ( $\mu$ gees) & Velocity $(\mathrm{nm} / \mathrm{s})$ & Displacement (pm) \\
\hline \multirow[t]{9}{*}{1} & 133 & 23 & Root & $\mathrm{x}$ & 220 & 200 & 20 \\
\hline & & & & $\mathrm{y}$ & 320 & 200 & 25 \\
\hline & & & & $\mathrm{z}$ & 320 & 400 & 50 \\
\hline & & & Middle & $\mathrm{x}$ & 80 & 100 & 10 \\
\hline & & & & $\mathrm{y}$ & 140 & 100 & 10 \\
\hline & & & & $\mathrm{z}$ & 190 & 150 & 15 \\
\hline & & & Tip & $\mathrm{x}$ & 60 & 60 & 7 \\
\hline & & & & $\mathrm{y}$ & 80 & 100 & 10 \\
\hline & & & & $\mathrm{z}$ & - & - & - \\
\hline \multirow[t]{5}{*}{2} & 301 & 32 & Root & $\mathrm{x}$ & - & - & - \\
\hline & & & & $\mathrm{y}$ & 80 & 80 & 9 \\
\hline & & & & $\mathrm{z}$ & 220 & 200 & 25 \\
\hline & & & Middle & $\mathrm{x}, \mathrm{y}, \mathrm{z}$ & - & - & - \\
\hline & & & Tip & $\mathrm{x}, \mathrm{y}, \mathrm{z}$ & - & - & - \\
\hline \multirow[t]{5}{*}{3} & 188 & 28 & Root & $\mathrm{x}$ & - & - & - \\
\hline & & & & $\mathrm{y}$ & 50 & 60 & 7 \\
\hline & & & & Z & 125 & 150 & 17 \\
\hline & & & Middle & $\mathrm{x}, \mathrm{y}, \mathrm{z}$ & - & - & - \\
\hline & & & Tip & $\mathrm{x}, \mathrm{y}, \mathrm{z}$ & - & - & - \\
\hline \multirow[t]{9}{*}{4} & 284 & 31 & Root & $\mathrm{x}$ & - & - & - \\
\hline & & & & $\mathrm{y}$ & - & - & - \\
\hline & & & & $\mathrm{z}$ & 130 & 150 & 15 \\
\hline & & & Middle & $\mathrm{x}$ & - & - & - \\
\hline & & & & $\mathrm{y}$ & - & - & - \\
\hline & & & & z & 75 & 100 & 12 \\
\hline & & & Tip & $\mathrm{x}$ & - & - & - \\
\hline & & & & $\mathrm{y}$ & 62 & 50 & 7 \\
\hline & & & & z & - & - & - \\
\hline
\end{tabular}

In these methods, the data is enveloped in specified frequency bands, and anomalies are identified as statistically significant deviations above the observed background persistent vibration, some of which is noise, some of which is real vibration. Thresholds of 3 to 5 standard deviations have proven successful in detecting unambiguous spontaneous vibrations.

In this analysis, nearly $6000 \mathrm{~s}$ of data was collected (approximately $2.3 \mathrm{G}$ Bytes). An initial visual inspection of the unfiltered data detected spontaneous vibrations on the order of $0.4 \mathrm{~s}$ long with amplitudes greater than 7 standard deviations. To ensure a thorough analysis, the data was autonomously scanned using the following protocol.

Three band-pass filters were applied to all of the Boom-mounted accelerometer data. The filter ranges were $200-500 \mathrm{~Hz}, 500-1000 \mathrm{~Hz}$, and $1000-2300 \mathrm{~Hz}$. After filtering, the value for standard deviation was computed for each $13.1 \mathrm{~s}$ data window. In each window, a threshold level of 7 standard deviations was used for event detection. For a Gaussian distribution, the expected probability of having a $7 \sigma$ result in a random data set is $2.6 \mathrm{e}-10 \%$. In these tests, results greater than 7 standard deviations occurred in $2 \mathrm{e}-5 \%$ of the samples. That relates to 77,000 times greater occurrence of 7 standard deviation events than is statistically probable.

Finally, each event above this threshold was visually compared with the corresponding table vibration data. This is a final check, which eliminates exogenous vibration sources. No measured input from the environment was found in any identified spontaneous vibration event.

\section{Results}

A total of six thermal radiation loads were applied. The results indicated the presence of four spontaneous vibrations. These are listed in Table 2 . The velocity and displacement amplitudes were determined by narrow-band integration of the accelerometer signals.

Once a possible anomaly was detected, it was further interrogated by temporal and spectral analysis. This is described further in the context of individual results in the section that follows. 




Fig. 2. Temperature Profile for Events 1 and 2.



Fig. 3. Accelerometer Response during Thermal Excitation Period at Node 3004 in the Z-Direction (High-pass Filtered above 100 Hz).

The temperature profile, during which two of the four events were detected, is shown in Fig. 2. The thermal lamps were on for the time period between 0 seconds and 300 seconds. The overall temperature change at the temperature gauge nearest the lamps was $32.5^{\circ} \mathrm{C}$. (Notice also the duty cycle of the data windows, which indicates the 20-second data recording time needed between data acquisitions.) In Fig. 2 and all other data plots and times reported in this paper, the time at which the thermal lamps were turned on is considered zero seconds.

Figure 3 plots the sequence of data for one of the measurement channels during the heating period. Using the 

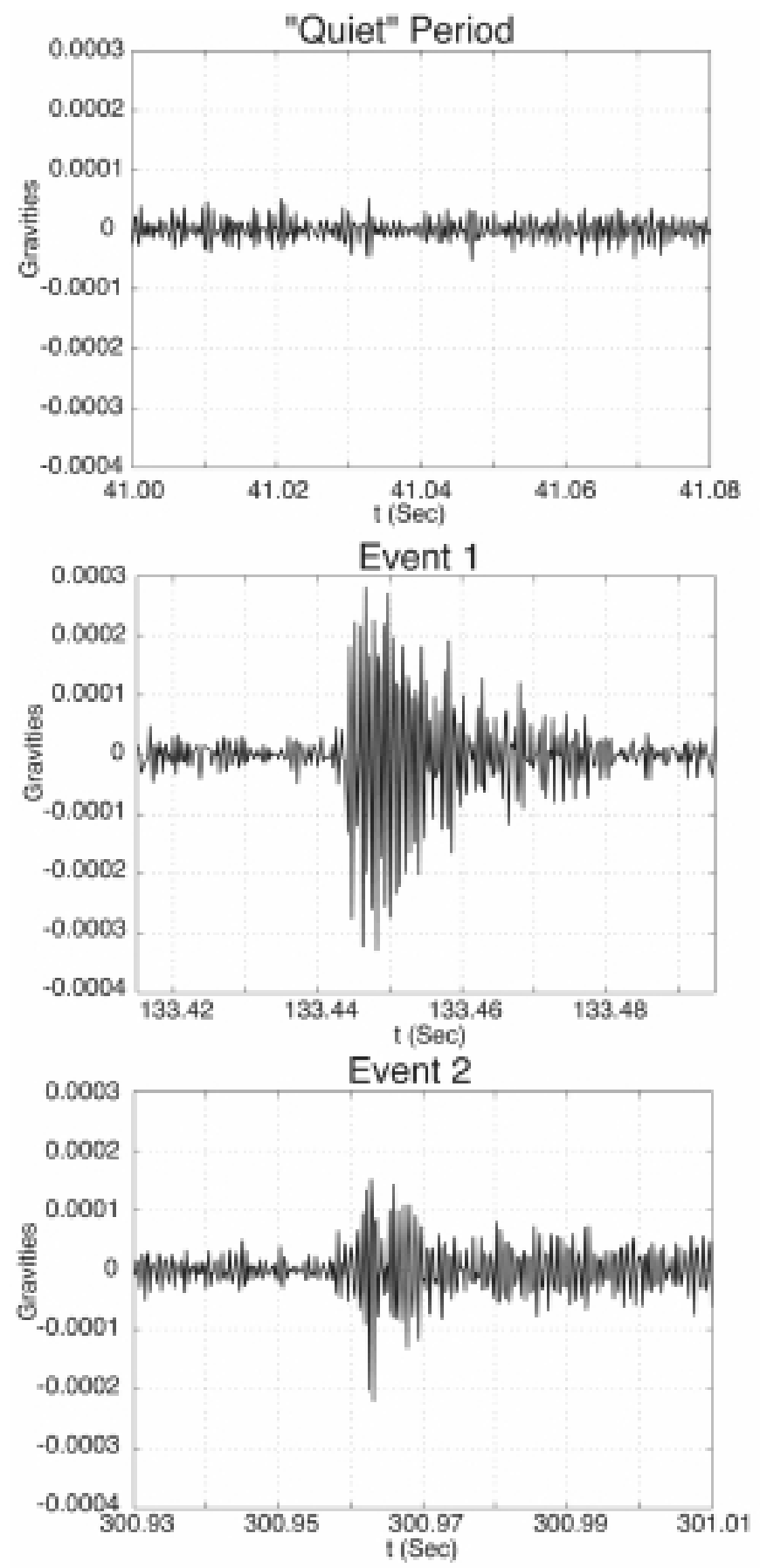

Fig. 4. Detail of Accelerometer Response during Thermal Excitation Period at Node 3004 in the Z-Direction (High-pass Filtered above 100 Hz).

above protocol, two dynamic events were identified during this period, designated Event 1 and Event 2 . It should be noted that the persistent motion in this data set is not sensor noise, but vibration of the boom. A detail of a period of persistent motion, or "quiet" period, along with details of Events 1 and 2 are shown in Fig. 4. The data shown in Figs 3 and 4 are band-pass filtered between 1100 and $2000 \mathrm{~Hz}$.

A comparison of results for Event 1 from Fig. 3 at different parts of the boom is shown in Fig. 5. Figure 6 shows 
Table 3

$1500 \mathrm{~Hz}$ Excitation Frequency Comparison

\begin{tabular}{llc}
\hline Section & Mode Type (Clamped) & Mode No. Nearest to $1500 \mathrm{~Hz}$ \\
\hline Batten & Transverse & 12 \\
& Longitudinal & $\ll 1$ \\
\multirow{2}{*}{ Longeron } & Transverse & 10 \\
& Longitudinal & $\ll 1$ \\
Diagonal & Drum Mode & $\gg 38$ \\
\hline
\end{tabular}
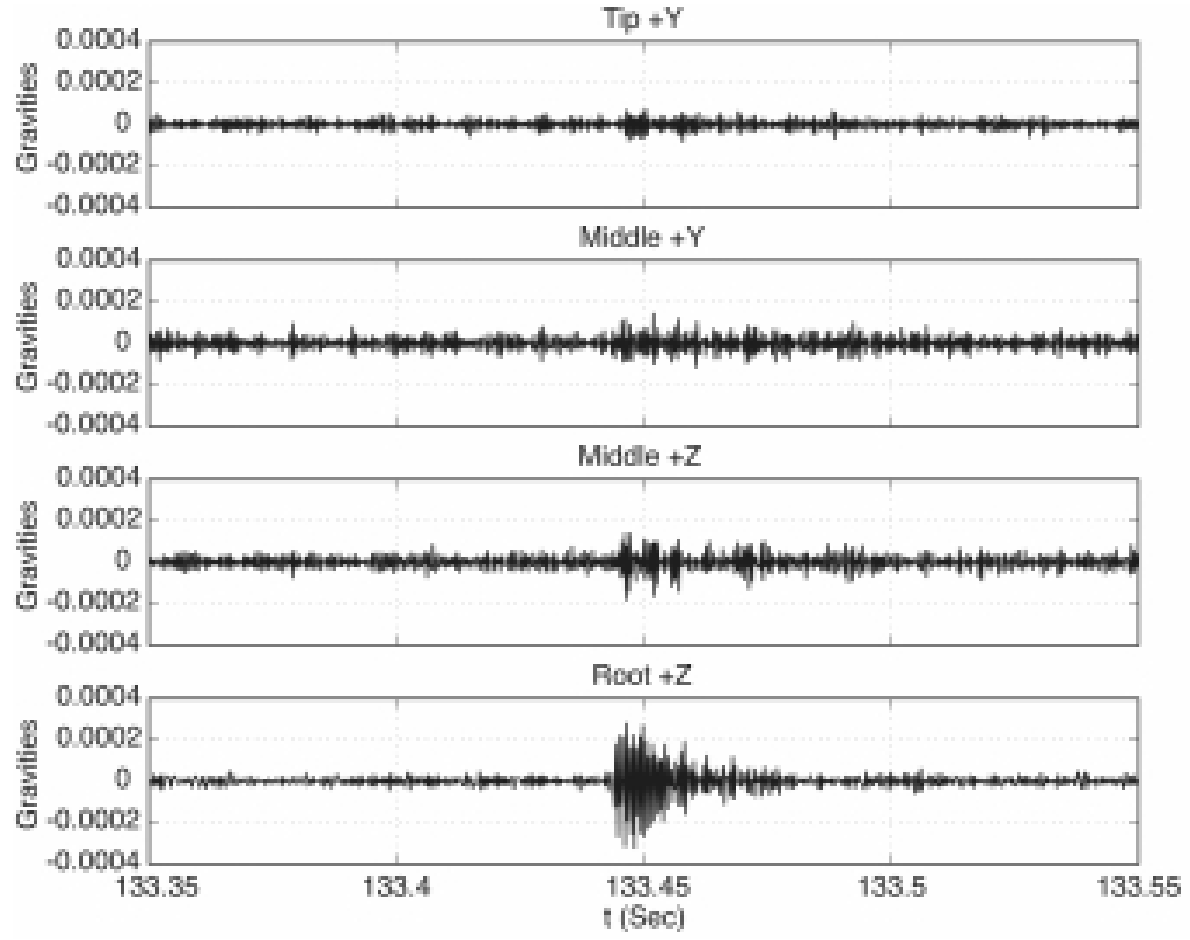

Fig. 5. Response Dispersion of Event 1 along the Boom (Band-pass Filtered between 1.1 and $2 \mathrm{kHz}$ ).

the spectrogram of this time data in a similar time window. Two key characteristics of the response can be noted in this comparison. First, Figs 5 and 6 show the propagation of the response along the boom. The response is larger in magnitude close to where the thermal load was applied near the root of the boom (3004), at the middle and tip of the boom (7003 and 10001 respectively), the response magnitude decreases. The peaks in the spectrogram of Fig. 6 occur a few milliseconds later at the tip (10001) compared to the root (3004). Using a threshold of $4 \sigma$ to detect the start of the response in the data at the middle and tip shows that the response takes 2.0 and 2.8 milliseconds to propagate respectively. Second, these figures show the directional change of the response as it travels down the boom. For this particular event, the response direction changes from mainly in the z-direction at the root to mainly in the y-direction at the tip. Third, the spectrogram also shows that the frequency content of the Event is different in each sensor, indicative of wave dispersion and perhaps nonlinear transmission through the structure. The variation in the response to a spontaneous vibration with respect to direction and frequency content in each direction at each point of measurement can be explained due to the structural complexity of the truss.

The appendix presents plots of all the time data and spectrograms for all the Events and degrees of freedom identified in Table 2.

The dominant excited frequency at the root is $1500 \mathrm{~Hz}$. Table 3 compares this to the calculated local frequencies of components of the boom [4]. This value is nearest to the 10th transverse clamped mode of a longeron, or much greater than the theoretical 38th drum mode of the cable diagonals [4]. It is unknown what the precise distribution of vibration energy was during these events. Nonetheless, even though these theoretical component frequencies are 
Table 4

Comparison of Band-Limited RMS for Sliding Time Windows During and Following Event 1

\begin{tabular}{lcccc}
\hline Location & Direction & $\begin{array}{c}\text { Event 1 RMS } \\
(\mu \text { gees })\end{array}$ & $\begin{array}{c}\text { Mean Post-Event RMS } \\
(\mu \text { gees })\end{array}$ & $\begin{array}{c}\text { Max Post-Event RMS } \\
(\mu \text { gees })\end{array}$ \\
\hline Boom (3004) & $+\mathrm{X}$ & 48 & 10 & 23 \\
& $+\mathrm{Y}$ & 86 & 12 & 25 \\
\multirow{3}{*}{ Table (101) } & $+\mathrm{Z}$ & 171 & 13 & 32 \\
& $+\mathrm{X}$ & 2 & 2 & 5 \\
& $+\mathrm{Y}$ & 3 & 3 & 5 \\
\hline
\end{tabular}
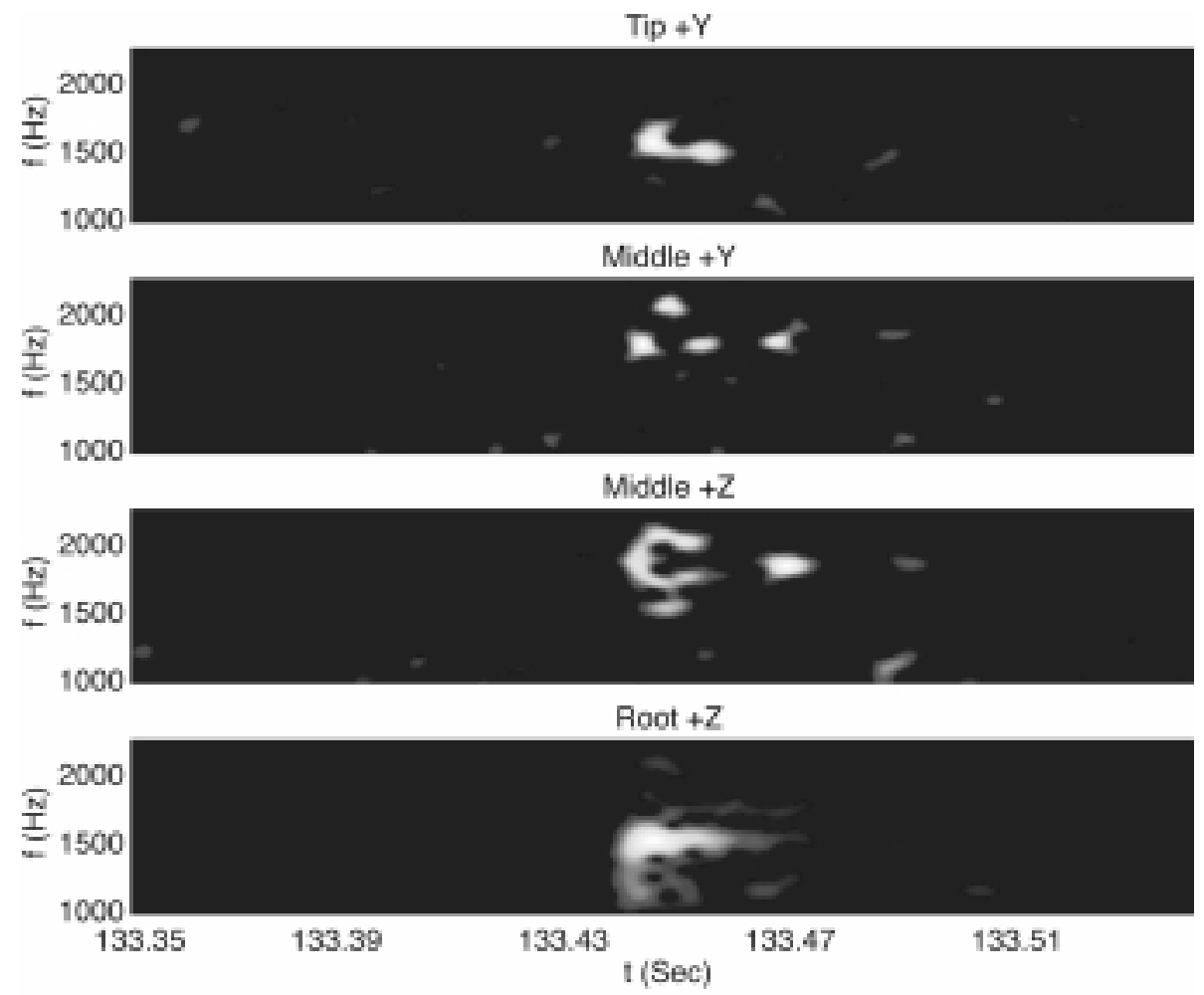

Fig. 6. Response Dispersion Spectrogram of Event 1 along the Boom.

well into the range where one would be suspect about their precise value the frequency seen in the experiments is so high compared with local modes that it might be considered more "acoustic" than "modal" [13].

\section{External source investigation and error analysis}

The first question one should ask upon seeing such a response in a structure is whether or not the response was an ordinary vibration induced by the environment. Making such a determination requires that the vibration in the boom be compared to the acceleration in the optics bench. Figure 7 compares three of the optics bench accelerometers to the strongest $(3004+Z)$ acceleration measurement on the boom. As can be seen, the relatively large 300 micro-gee vibration of the boom is not visually correlated with a corresponding event in the table.

To make a quantitative comparison, a band-limited RMS for sliding time windows was computed for each measurement. The RMS was limited to a frequency bandwidth of 1200 to $1800 \mathrm{~Hz}$, to bracket the frequency content of the event. Each window was 0.01 seconds in length, which was approximately the duration of Event 1. Table 4 presents the results of this analysis. It compares the RMS of each measured channel in Fig. 7 during 




Fig. 7. Response at Node 3004 of Event 1 Compared to Corresponding Table Accelerometer Data (High Pass Filtered above $100 \mathrm{~Hz}$ ).

the single window of Event 1 to the 12.5 seconds of data following Event 1. During Event 1, the RMS in all three boom-mounted accelerations is much higher than the average or maximum RMS in those channels. However, the RMS in the table accelerometers does not show a deviation outside the range measured in the post-Event 1 period. The RMS in the measurements following Event 1 can be taken to characterize the net measurement uncertainty or "noise," combining sensor resolution and environmental stability. With this definition, this data indicates that the boom vibration in Event 1 significantly exceeds the corresponding measurement uncertainties, while the table vibration stays within the measurement uncertainty.

Thus, there are two possibilities:

(a) The response is due to an internal mechanism in the boom, or

(b) The response is a transient amplification of an unresolvable induced vibration with an amplification factor of at least 200 above the background vibration.

The latter possibility can be discounted with the following argument. Amplification like this might result from a harmonic resonance of the structure. But that would require, in this case, a dynamic amplification corresponding to $0.25 \%$ damping, which is much lower than the damping observed in the boom (typically 1-5\% damping). Also, the response is short-lived, less than 3 cycles to peak amplitude, which is also inconsistent with the notion that it is a harmonic resonance. Finally, as seen in the spectrogram of Fig. 6, the frequency content is not mono-modal but consists of several modes, the frequencies of which seem to shift with location along the boom. All measurements of small-scale dynamic behavior will ultimately be left with this level of uncertainty: i.e., "Was the response something ordinary to some input that was unmeasured?" But in this case, such doubt does not seem to be supported by the data. Moreover, there were no spontaneous vibrations detected during the times before the application of heat, meaning that the vibrations were always associated with the temperature rise in the boom. All these factors indicate that the vibrations were due to an internal mechanism within the boom. 
Causality analysis is an analytical method that is often employed in finding the vibration sources and it is very useful in structures with either a few vibration transmission paths or a continuum of transmission paths. However, for structures with a large, finite number of transmission paths, the analysis technique becomes excessively ambiguous, and hence not useful [17]. The IPEX boom contains many battens, longerons and tensioned diagonals making a large but finite number of transmission paths. Therefore, a causality analysis of the vibration data would most likely not be able to determine whether or not the vibrations occurred spontaneously from within the boom.

\section{Conclusions}

This paper reported observations of picometer scale spontaneous vibrations in a precision deployable boom under thermal loading. The structural test article was the IPEX-2 deployable boom previously flown in space. In an attempt to produce the spontaneous vibrations that were seen in orbit, the test article was thermally loaded within a stabilized test environment. Spontaneous vibrations were induced in these experiments. The amplitudes of these vibrations were on the order of a few dozen picometers, and the frequency was near $1500 \mathrm{~Hz}$. Evidence of wave dispersion was demonstrated. The analysis of the test data is inconclusive as to whether the spontaneous vibrations resulted from a release of friction in a mechanism or from material anelastic instability. In either case, the vibrations were at a scale of motion for which current theories would not expect such a release. An analysis of the measurement error and possible external vibration sources was also presented concluding that the measured vibrations did in fact come from within the boom. Further work should consider whether friction laws ordinarily applied at macroscopic scales of deformation should be reconsidered at such small scales of motion.

\section{Acknowledgements}

This research was sponsored by NASA Jet Propulsion Laboratory contract 960896, with Dr. Marie Levine as Technical Monitor.

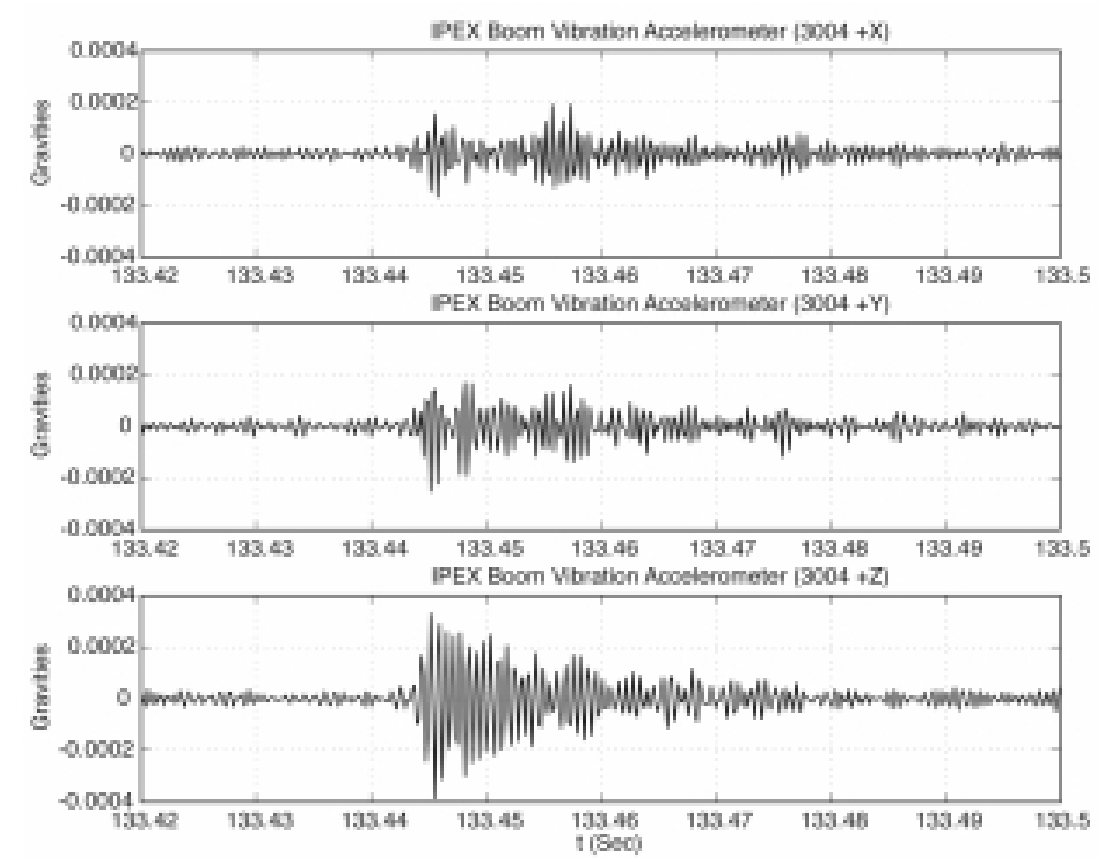

Fig. A1. Event 1 Node 3004. 

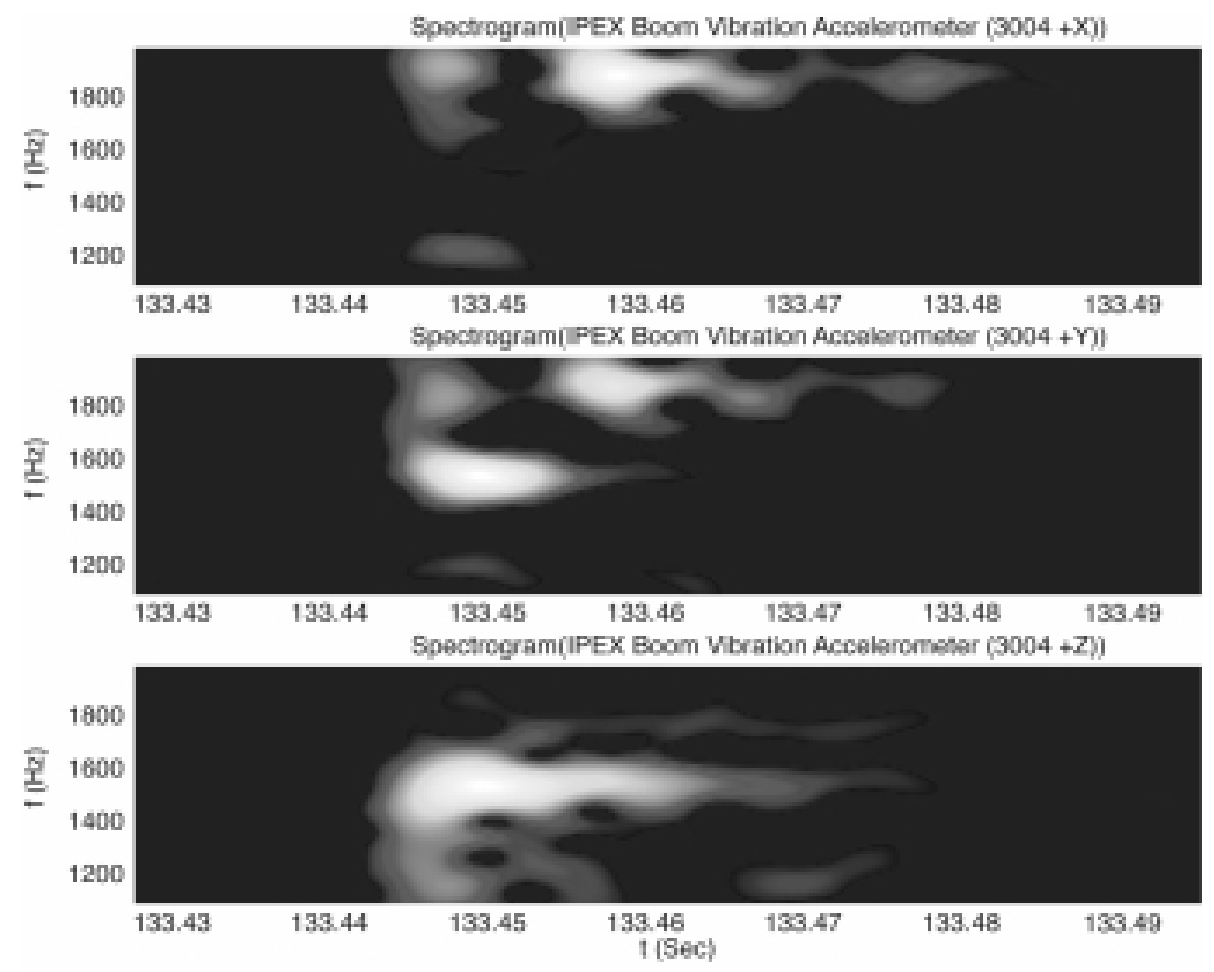

Fig. A2. Event 1 Node 3004 Spectrogram.


Fig. A3. Event 1 Node 7003. 


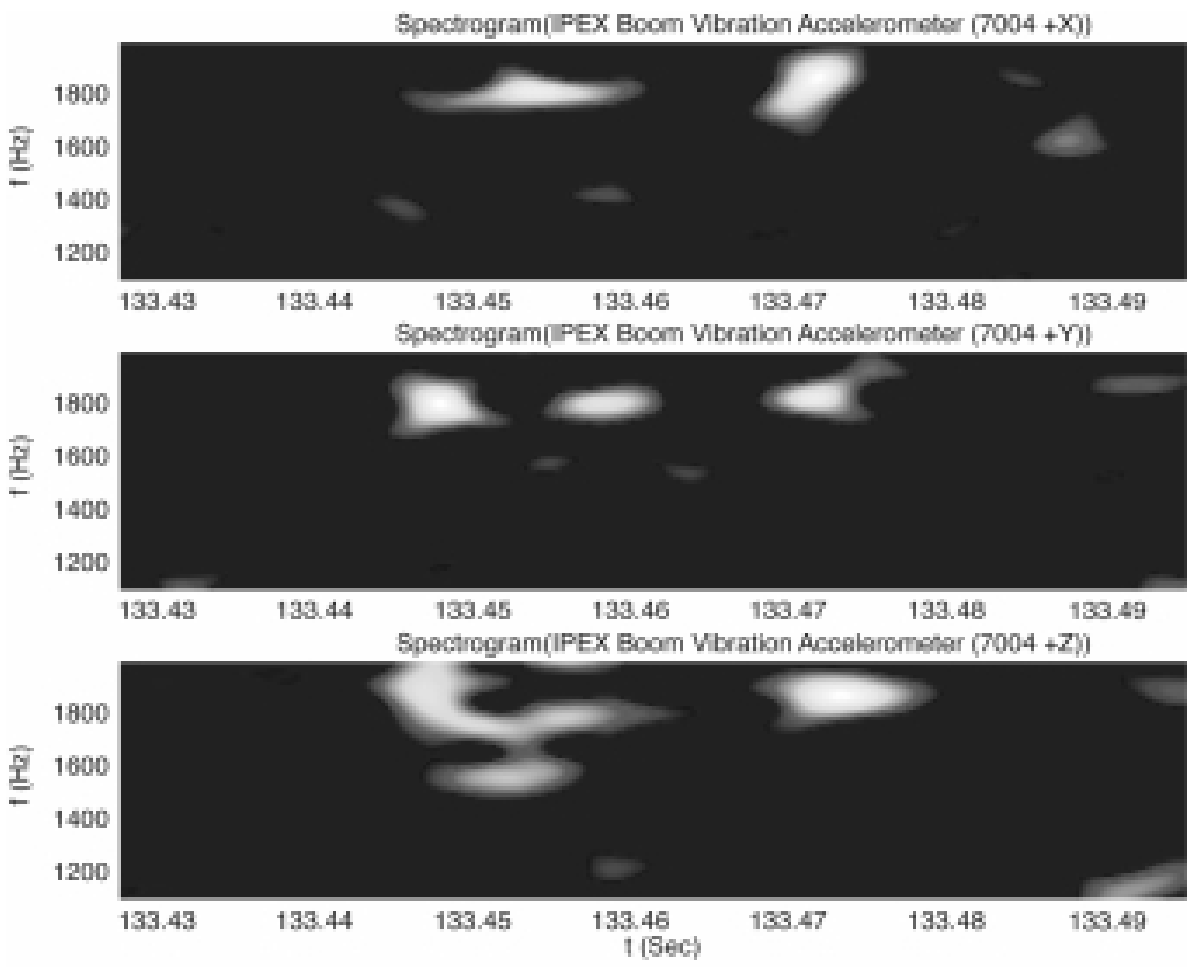

Fig. A4. Event 1 Node 7003 Spectrogram.
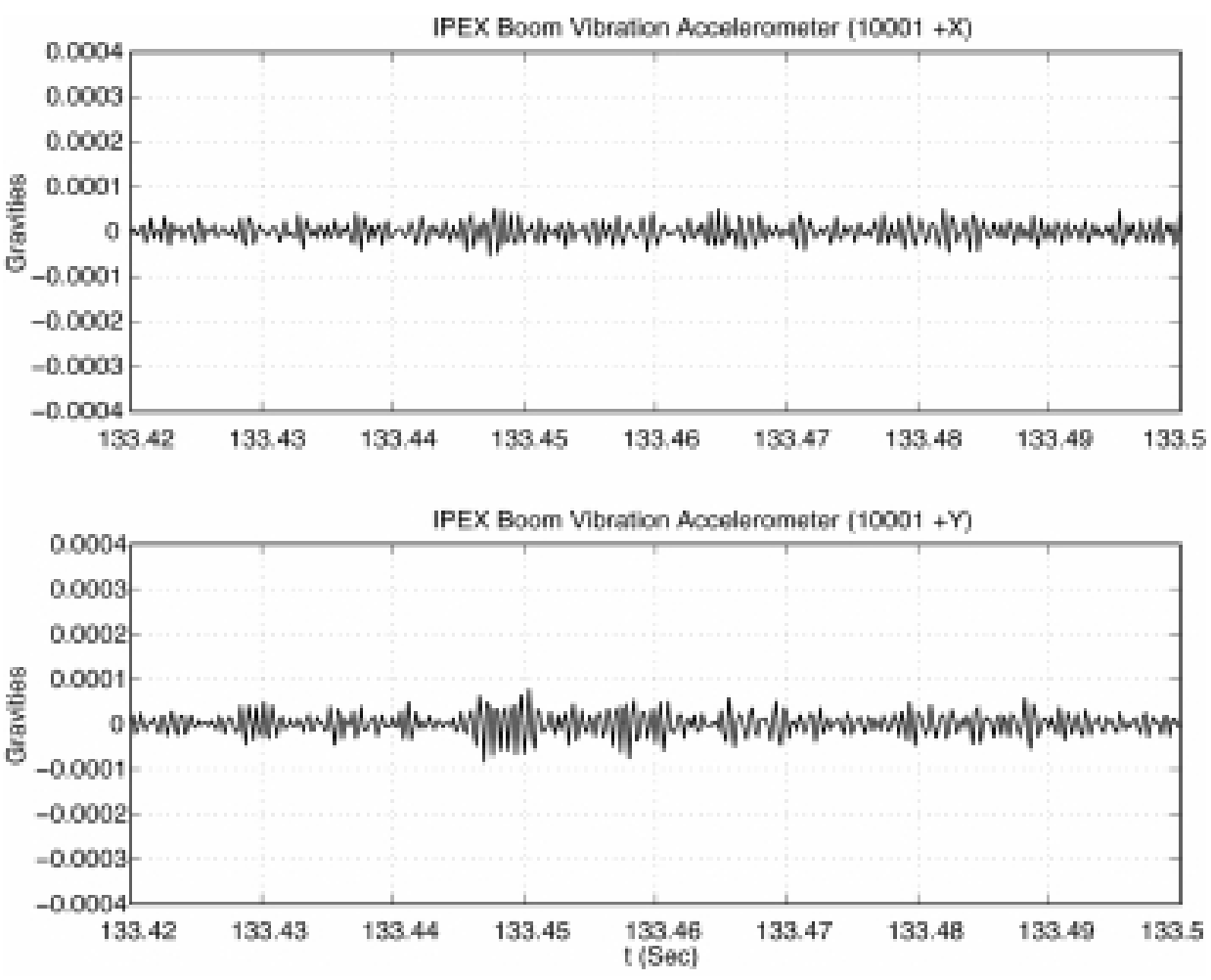

Fig. A5. Event 1 Node 10001 

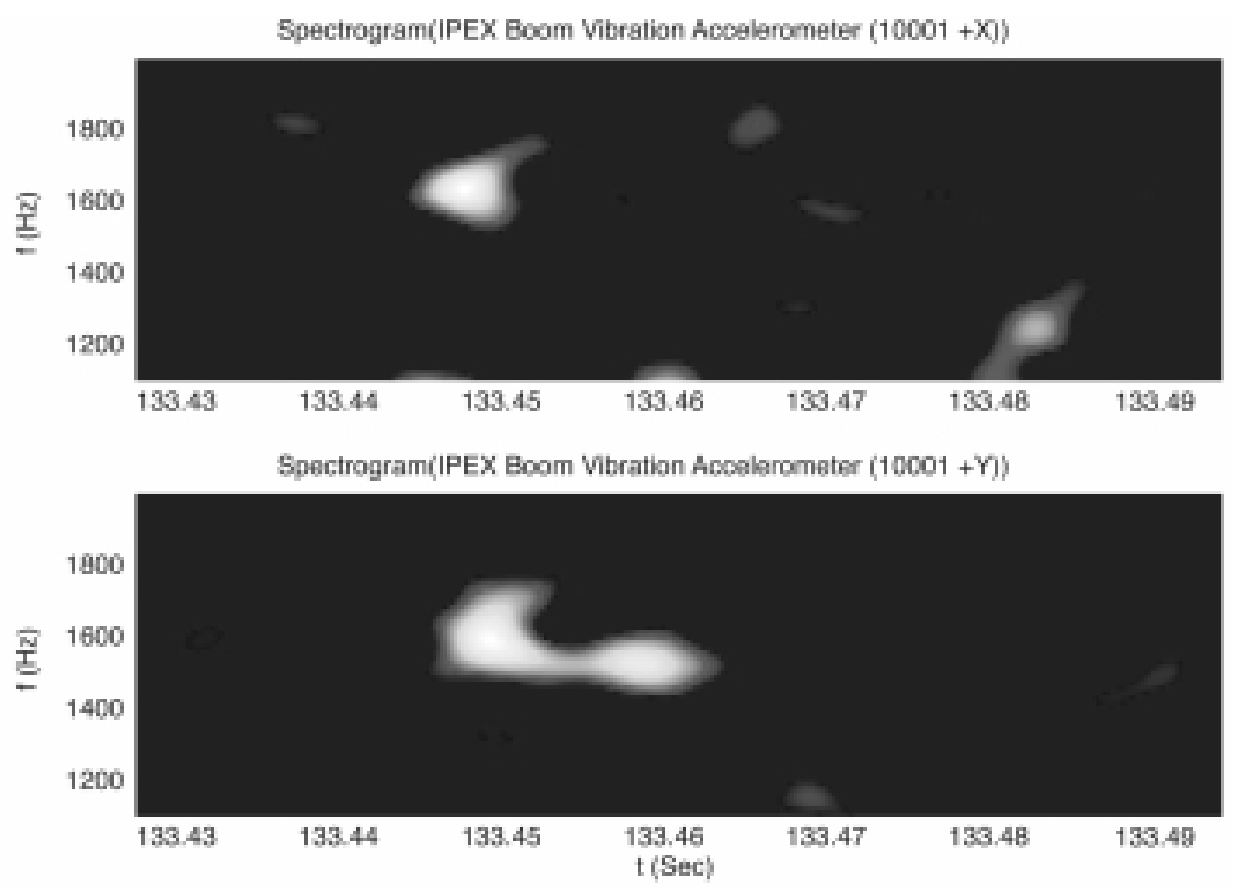

Fig. A6. Event 1 Node 10001 Spectrogram.


Fig. A7. Event 2 Node 3004. 



Fig. A8. Event 2 Node 3004 Spectrogram.
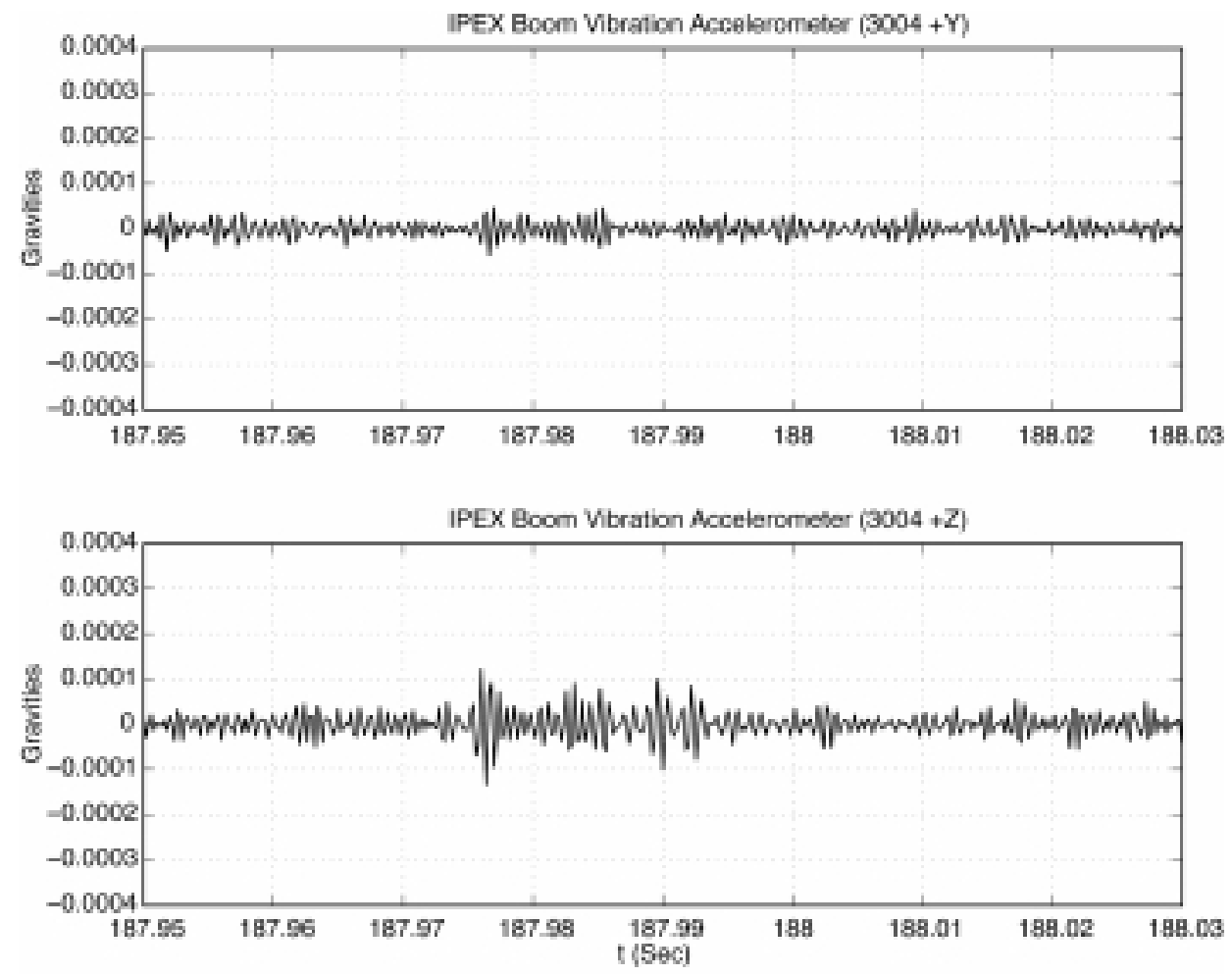

Fig. A9. Event 3 Node 3004. 

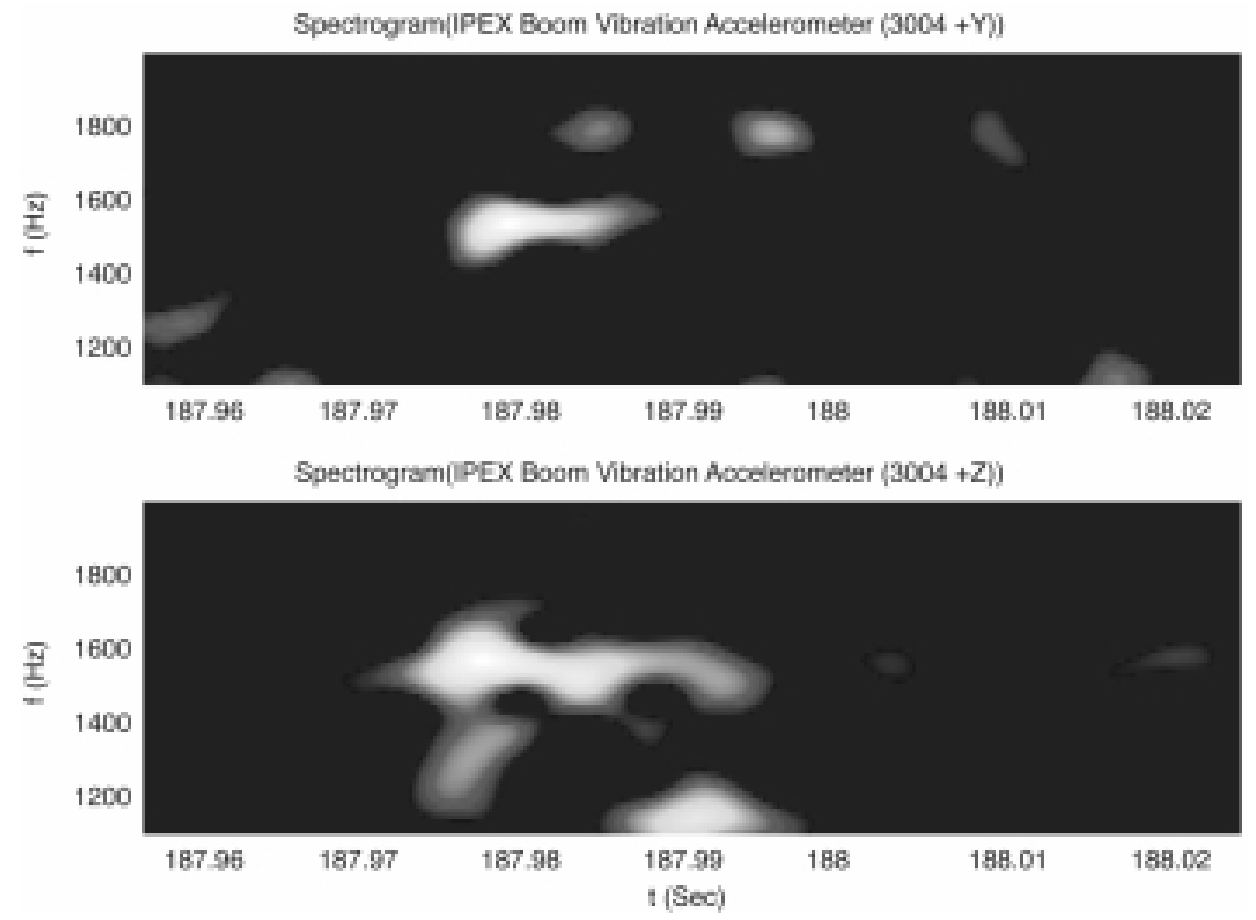

Fig. A10. Event 3 Node 3004 Spectrogram.


Fig. A11. Event 4 Nodes 3004, 7003 \& 10001. 




Fig. A12. Event 4 Nodes 3004, 7003 \& 10001 Spectrogram.

\section{References}

[1] B. Armstrong-Helouvry, P. Dupont and C. Canudas de Wit, A Survey of Models, Analysis Tools and Compensation Methods for the Control of Machines with Friction, Automatica 30(7) (1994), 1083-1138.

[2] J.F. Bell, Mechanics of Solids, Vol. I, Art. Intro, 2.27, 3.44, Springer-Verlag, 1984.

[3] P.-Y. Bely, The NGST Yardstick Mission, Proceedings of the 34th Liege International Astrophysics Colloquium, Liege, Belgium, June $15-18,1998,159-166$

[4] R.D. Blevins, Formulas for Natural Frequency and Mode Shape, Krieger Publishing, 1979.

[5] R. Danner and S. Unwin, Space Interferometry Mission: Taking the Measure of the Universe, http://sim.jpl.nana.gov/library/book.html, March 1999.

[6] C.L. Foster, M.L. Tinker, G.S. Nurre and W.A. Till, The Solar Array-Induced Disturbance of the Hubble Space Telescope Pointing System, NASA-TP-3556, Marshall Space Flight Center, AL, May, 1995.

[7] L.M.R. Hardaway, Stability and Mechanics of Precision Deployable Structures Under Nanometer Deformation, Ph.D. Dissertation, University of Colorado, 2000.

[8] L.M.R. Hardaway and L.D. Peterson, Nanometer Scale Spontaneous Vibrations in a Deployable Truss Under Mechanical Loading, AIAA Journal 40(10) (October 2002), 2070-2076.

[9] M. Ingham, E. Crawley and D. Miller, Microdynamics and Thermal Snap Response of Deployable Space Structures, SERC \#2-98, May 1998.

[10] M. Ingham and M. Levine, IPEX-2 Flight Data Analysis: Quiescent Period Report, NASA JPL D-17912, June 30, 1999.

[11] K.L. Johnson, Contact Mechanics, Cambridge University Press, 1985.

[12] G. Ortiz, M. Levine and C.Y. Peng, IPEX-2 Post-Flight Ground Modal Test Report, NASA JPL D-19383, July 21, 2000.

[13] J.R. Mathews, Introduction, in: Acoustic Emission, J.R. Matthews, ed., Gordon and Breach Science Publisher, NY, 1983.

[14] C.Y. Peng, Assessment of Thermal induced Joint Loads of IPEX-2 Boom, JPL 352F:99:029:CYP, November 22, 1999.

[15] L.D. Peterson and J.D. Hinkle, Microdynamic Design Requirements for Large Space Structures, AIAA-2003-1451, Proceedings of the 44th Structures, Structural Dynamics and Materials Conference, Norfolk, Virginia, April, 2003.

[16] E.A. Thornton, Thermal Structures for Aerospace Applications, AIAA, Reston, VA, 1996.

[17] P.H. White, Cross Correlation in Structural Systems: Dispersion and Nondispersion Waves, Journal of the Acoustical Society of America 45(5) (1969). 



Submit your manuscripts at

http://www.hindawi.com
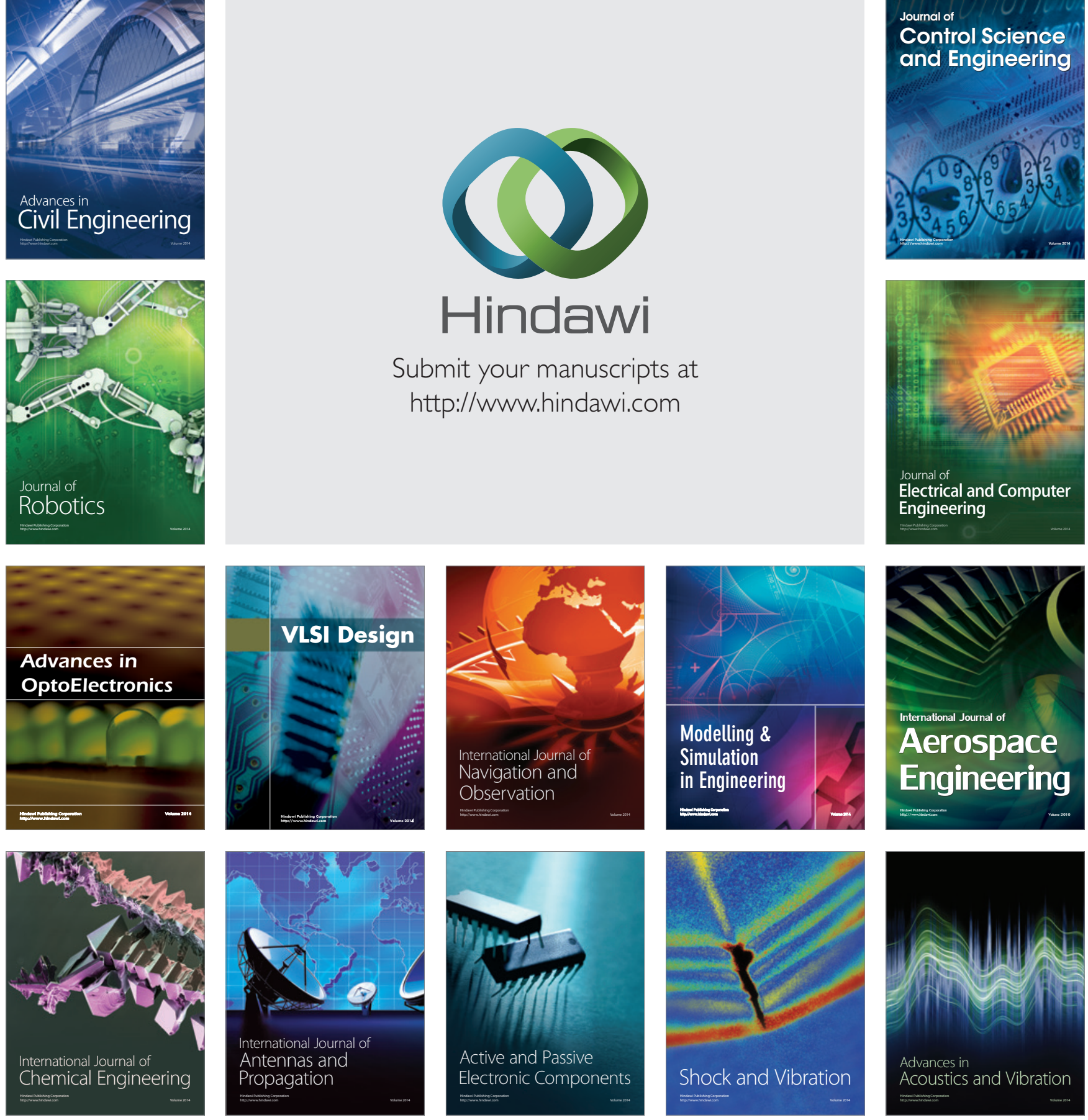